\title{
Bio-Memristor Based on Peptide and Peptide Composite with Gold Nanoparticles
}

\author{
Alexander Loskutov ${ }^{1 *}$ \\ ${ }^{1}$ Moscow State Technological University "STANKIN", RU-127055, Moscow, Russia
}

\begin{abstract}
The structure, morphology and electrical properties of thin dipeptide hexamethylenediamide bis (N-monosuccinylglutamlysin) (DPT) layers and a DPT composite with gold nanoparticles deposited on gold and HOPG substrates were studied by probe microscopy and spectroscopy. The chemical formula of DPT is: $\left\{\mathrm{HOOC}-\left(\mathrm{CH}_{2}\right)_{2}-\mathrm{CO}_{-}{ }_{\mathrm{L}}-\mathrm{Glu}^{-}{ }_{\mathrm{L}}{ }^{-}\right.$ Lys-NH- $\left.\left(\mathrm{CH}_{2}\right)_{3}\right\}_{2}$, and it is a mimetic of nerve growth factor. The results demonstrate that the structure and morphology of DPT thin layers depend significantly on the molecule charge (neutral or anion) and the nature of the substrate-layer interface. It was possible to control the structure and properties of the formed solid layers by changing $\mathrm{pH}$ of aqua solution (the charge of the DPT molecule). Bipolar resistive switching was observed in thin DPT layers on graphite and gold surfaces. The crystallization of anions on the surface of gold led to the formation of a ferroelectric unlike graphite. A strong dependence of the morphology of DPT composite layers on the nature of the substrate and the state of its surface is revealed. It indicates the important role of interfacial interactions in the crystallization processes of the DPT layers. The electrical properties of layers also depend on the interaction of DPT with the substrate. An increase in the thickness of the layers significantly affects the morphology and value of the tunneling current. Similar to crystallization of DPT salt on a gold surface, crystallization of DPT composite with gold nanoparticles also leads to the formation of a ferroelectric. The differences found in the structure of DPT composite layers on graphite and gold surfaces can be explained by assuming that the structure of the second and all subsequent layers is completely determined by the structure of the first adsorption layer in DPT-substrate interface. So this layer serves as a template for the growth of all other layers. The results can find practical application in 3D printing technologies. The presence of negative differential conductivity on local tunnel currentvoltage characteristics of peptide composites is of great practical importance when used as active elements for amplifying current and power, memory cells in organic electronics. Investigated DPT has rather good memristive characteristics, including good endurance, satisfying ON/OFF current ratio, long retention time and reproducible write-once read-many times (WORM) memory behavior. All this allows us to consider the DPT to be a perspective material of memristor organic electronics. Since it is also a drug, the polymorphism and its dependence on $\mathrm{pH}$ can also find application in the pharmaceutical industry.
\end{abstract}

\section{Introduction}

Organic semiconductors have long attracted the attention of scientists and manufacturers of various electronic devices and components. It is suggested that they can complement classic inorganic electronics in several areas (for example, photoelectric converters, sensors, displays, memristors, LEDs, etc.). Currently, various organic electronic devices have been developed and massproduced: light-emitting diodes, field effect transistors, etc. They have significant advantages: lower cost, light weight, flexibility and diverse functionality, as well as the most suitable for large-scale industrial production based on liquid-phase technologies in combination with inkjet printing methods and the ability of molecules to self-assemble. However, to obtain traditional organic compounds, it is necessary to use a fairly complex chemical synthesis. Biomolecular materials are free of this drawback, as they have their own structure, high environmental friendliness and biodegradability. A number of biomolecular materials, including DNA and peptides (PTs), have memristor properties [1-3]. Artificially synthesized PTs have a good ability to bind to the surfaces of inorganic materials, which allows them to be used for the synthesis of various nanostructures and coatings. [4-7].

Recently, fundamentally new opportunities have begun to appear for creating materials with desired functional properties. First of all, this is due to the development of nanotechnology and the emergence of

e-mail: ailoskutov@yandex.ru 
the task of molecular design. A wide variety of organic molecules and the possibility of a targeted change in their physical and electrical properties make it possible to use methods of molecular design to obtain functional solid layers with predetermined properties. It is also possible to customize the properties of such materials, for example, the photonic band gap, electronic and optical properties.

Molecular engineering is the synthesis of molecules with desired functional properties. But the functional properties of a substance are most often set at the macroscopic, and not at the molecular level. However, the relationship between the structure of molecules and supramolecular organization is usually very complex and often not studied. Therefore, the aim of establishing the relationship between the structure of an element and the form or properties of a macro system is of great scientific and practical importance. No less important are the processes of of self-assembly and self-organization of molecules and nanoparticles (NPs) due to the formation of intermolecular non-covalent bonds [8].

Non-volatile organic memory devices based on memristors can serve as promising elements of organic electronics [9, 10]. Memristor opens the way to the world of Boolean and non-Boolean applications, ranging from terabit non-volatile memory devices, algorithmic memory chips in logic and systems based on artificial neural networks created on the basis of biological data $[11,12]$. Very important, the hardware implementation of neuromorphic computing has been developed using the capacitive behavior observed in the memristor. [1315]. A capacitive feature presented today has been believed to be a unique stage of the memristor.

Previously, we investigated amyloid type PTs [4,5], which can be used not only in medicine and pharmacology, but also to create new solid-state advanced smart materials for organic microelectronics and devices based on them. These studies have shown that PT composite materials and coatings with silver and gold NPs do have a variety of structural, electrical and tribological properties that make them promising for large-scale practical applications in engineering. The influence of the conditions for the synthesis of gold NPs in aqueous solutions of a short dipeptide (DPT) on the architectonics of composite layers was investigated in [6, 7]. It was discovered that due to the specific zwitterionic structure of a PT molecule for the successful synthesis of gold nanoparticles (NPs), it was principally necessary to increase the $\mathrm{pH}$ of solution and, consequently, to increase the net negative charge of the molecule. Moreover, the structure of the molecule itself and the structure and morphology of the formed DPT layers are seriously altered. The nature of the substrate also affected the structure and morphology of the DPT layers. For the present work we used the same DPT. But unlike the previous work, the aim of the work is to investigate the mechanism of influence of these factors, including method of deposition, on the crystallization processes of thin DPT layers, to determine the structure-property relationship of the formed layers and the feasibility of practical application of DPT in engineering. In the latter case, it is necessary to reveal a relationship between morphology and electronic energy structure of the formed DPT layers on solid surfaces. To elucidate the structure-property interrelation, a high level of understanding of the underlying processes and mechanisms is required. All this requires more detailed studies of all stages of the processes of DPT crystallization and the influence of various factors on them.

In this paper, electrical and structural properties of composite peptide layers with gold NPs are considered with the perspective of using them as functional materials of organic electronics.

\section{Experiment.}

A DPT of hexamethylenediamide bis (Nmonosuccinylglutamlysin) was utilized. It belongs to the class of short neuroprotection PTs and is a mimetic of nerve growth factor [16]. Its chemical formula is: \{HOOC-(CH2)2-CO-L-Glu-L-Lys-NH-(CH2)3\}2, molecular weight $\mathrm{Mw}=830.9 \mathrm{Da}$.

The experiments were carried out at $\mathrm{pH}$ values of 4,2 (neutral molecule) (DPT-0) and 7,4 (anion) (DPT-1, DPT-nAu). An increase in $\mathrm{pH}$ was obtained by adding potassium carbonate to aqua solution. The choice of such high $\mathrm{pH}$ values was due to the fact that DPT- 1 is a precursor for the one-pot synthesis of gold NPs [6]. In each experiment, $10 \mathrm{ml}$ of DPT aqueous solution at different $\mathrm{pH}$ was deposited at room temperature on the polycrystalline gold films and HOPG (ZYH) with misorientation angles of $3.5-5^{\circ}$ (NT-MDT, Russia) surfaces. Thin gold films were prepared by magnetron sputtering under vacuum at room temperature.

The deposited DPT layers were dried in air at room temperature. Two methods of deposition were used: drop casting and spin coating at 2000-3000 rpm. After complete evaporation of solvent, a drop of colloidal solution on a solid surface leaves a distinct ring-shaped stain ("coffee-ring" effect) [17]. The surface images of deposited coatings were obtained using a universal highvacuum atomic force microscope (AFM) and a scanning tunneling microscope (STM) "Solver HV-MFM" (NTMDT, Russia) on air. The electric characteristics of the PT layers were determined by scanning tunneling spectroscopy (STS) by measuring local tunnel currentvoltage characteristics (LTCVC) in STM.

\section{Results and Discussion.}

AFM images of a surface of DPT thin layers, deposited at $\mathrm{pH}=4,2$ on HOPG and gold (DPT-0) are presented in Figure 1. On the HOPG it forms a continuous smooth layer that completely covers the terraces between the steps (Figure 1a). On the surface of gold, the formation of various linear structures of micron sizes also begins atop of a layer consisting of tightly packed "pancakes," which can be seen at higher resolutions (left corner of Figure 1b). A continuous layer of DPT-0 consists of densely packed flat rounded NPs $\leq 100 \mathrm{~nm}$ in size with aspect ratio (height /diameter) $\sim 0.15$ (Figure $1 \mathrm{~b}$ ). It is interesting that the presence of defects on the graphite 
surface (steps, terraces) practically has no effect on their orientation and growth of DPT layers

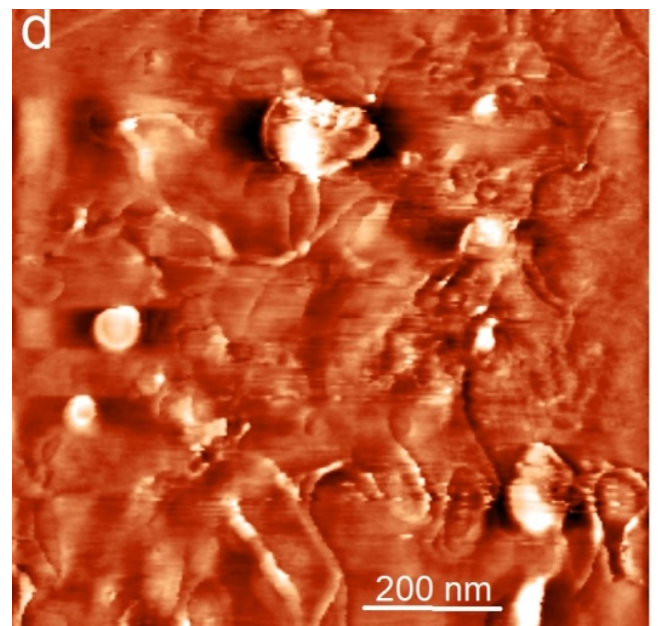

AFM images of thin layers of DPT-1 are presented in fig. 2.

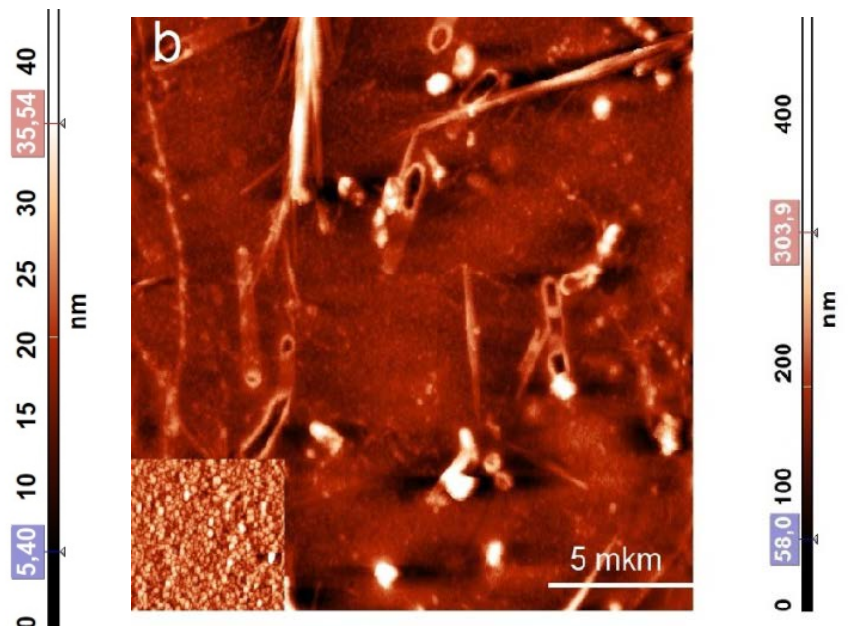

Fig. 1. AFM images of thin layers of DPT-0 on the surfaces of graphite (a) and gold (b).

Rather interesting morphology has seen in thin layers of DPT-1 is the growth of extended fibrous structures of different widths on the graphite surface (Fig. 2a). The formation of various mesocrystals oriented in two perpendicular directions occurs on the surface of gold atop a layer of densely packed particles (Figure 2b). At a higher resolution, it can be seen that these structures consist of strands with different widths. At low concentrations and ultrathin layers of DPT-nAu composite, gold NPs are clearly visible on the mica surface, which are surrounded by a ligand DPT shell (Figure 3a). The layers consist of gold NPs immersed in the DPT matrix. The thickness of the layer is negligible as evidenced by the presence of small pores. The average $\mathrm{NP}$ size is $50 \mathrm{~nm}$ and the height is less than $2 \mathrm{~nm}$ which results in the aspect ratio of 0.04 .
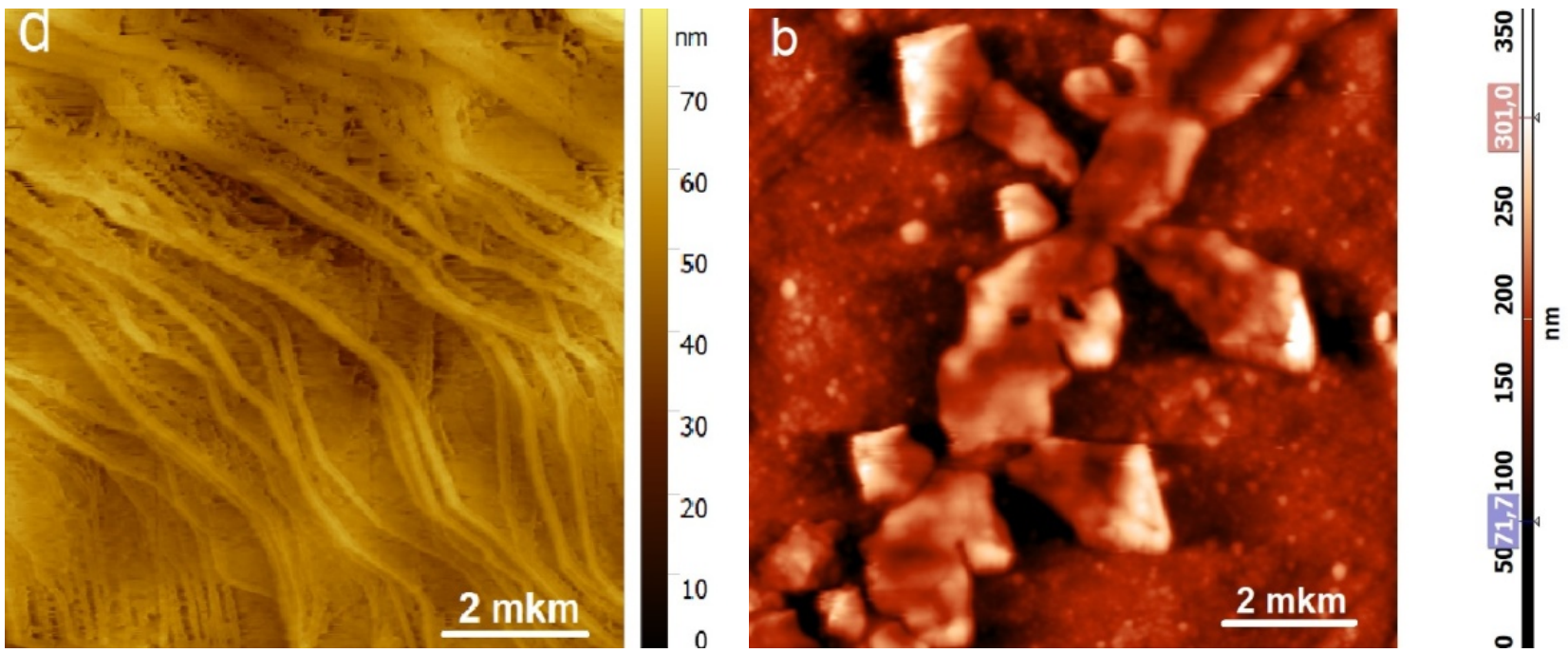

Fig. 2. AFM-STM images of thin layers of DPT-1 on the surfaces of graphite (a) and gold (b).

These data show that gold NPs have a shape of very thin flat discs, rather than spheres. Disordered multilayer structures consisting of separate flat particles of different sizes are formed on the surface of gold (Figure $3 \mathrm{~b}$ ). At earlier stages of thin layers growth, self-assembly of gold NPs occurs with the formation of separate linear chains consisting of 3 - 5 gold NPs. 

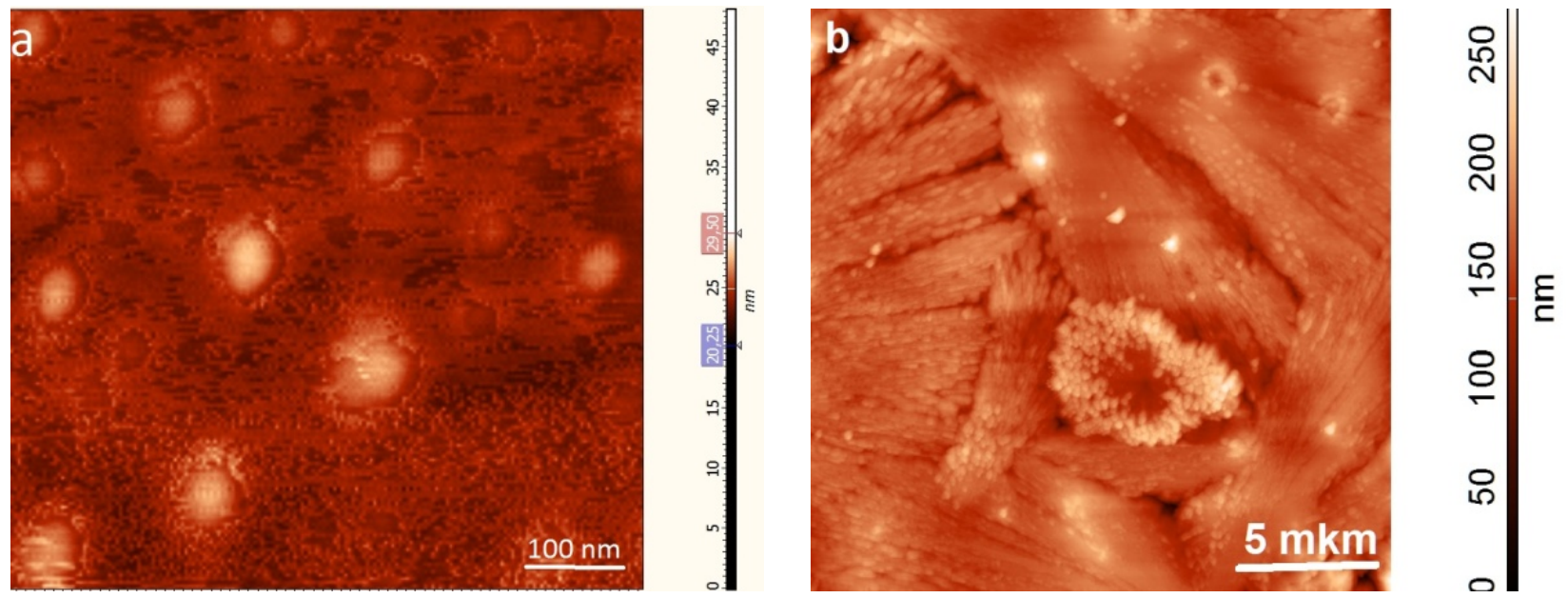

Fig. 3. AFM-STM images of ultra thin (a) and thin layers (b) of DPT-nAu on the surfaces of mica (a) and gold (b).

With increasing concentration, aggregation of composite NPs begins with the formation of threedimensional structures of various shapes without predominant orientation (Figure 3b). With an increase in the thickness of the layers to $\approx 1.5 \mu \mathrm{m}$, a gradual smoothing of the relief of the DPT-nAu layers is observed. At low concentrations and ultrathin layers of DPT-nAu composite, gold NPs are visible on the mica surface, which are surrounded by a ligand DPT shell (Figure 3a). The average NP size is $\sim 50 \mathrm{~nm}$, and the height is $<4 \mathrm{~nm}$. [6]. Given the data it follows that gold NPs are in the form of very thin disks, rather than spheres. At earlier stages of growth of thin layers, selfassembly of gold NPs occurs with the formation of separate linear chains consisting of 3 - 5 gold NPs [6].

On the HOPG substrate at the initial stages of DPT$\mathrm{nAu}$ growth the separate thin filaments and islands are formed (Figure 4a). They are oriented in different

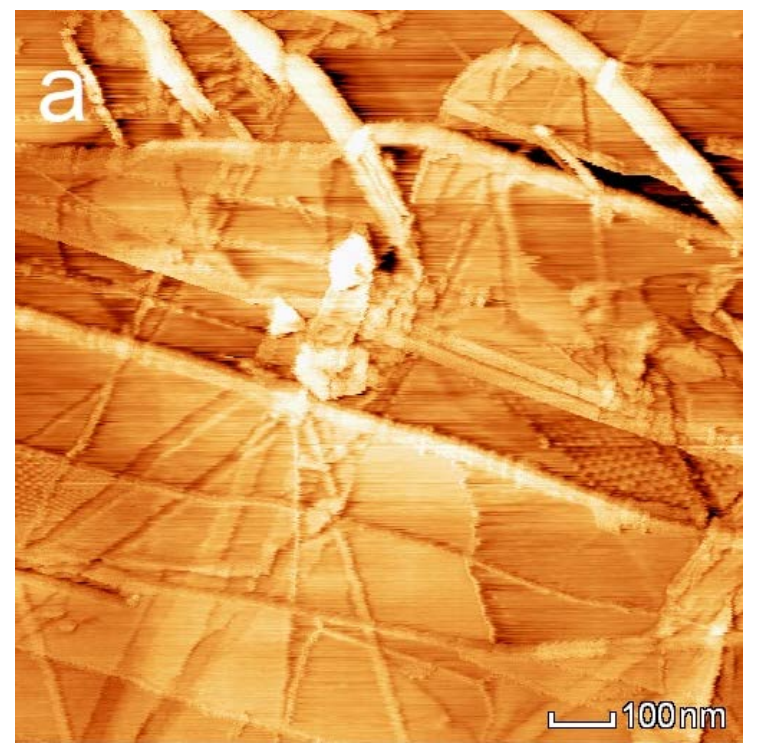

directions, regardless of the structure of the substrate surface. With an increase in the layer thickness, structures consisting of oriented stripes are formed (Figure 4b).

Local tunnel current-voltage characteristics (LTCVCs) at different points on the sample surface were measured to establish the relationship between the morphology of the surface of DPT layers and their electronic properties. The LTCVCs of DPT-0 and DPT-1 layers on gold and graphite were very different (Figure 5). Since in the STM measurements the probe characteristics remain constant, the change in the shape of LTCVCs on different samples is caused by a change in the local work function or the local density of electronic states of the substrate-DPT-tip system.

The most notable differences of LTCVCs shape were observed on the gold substrate.

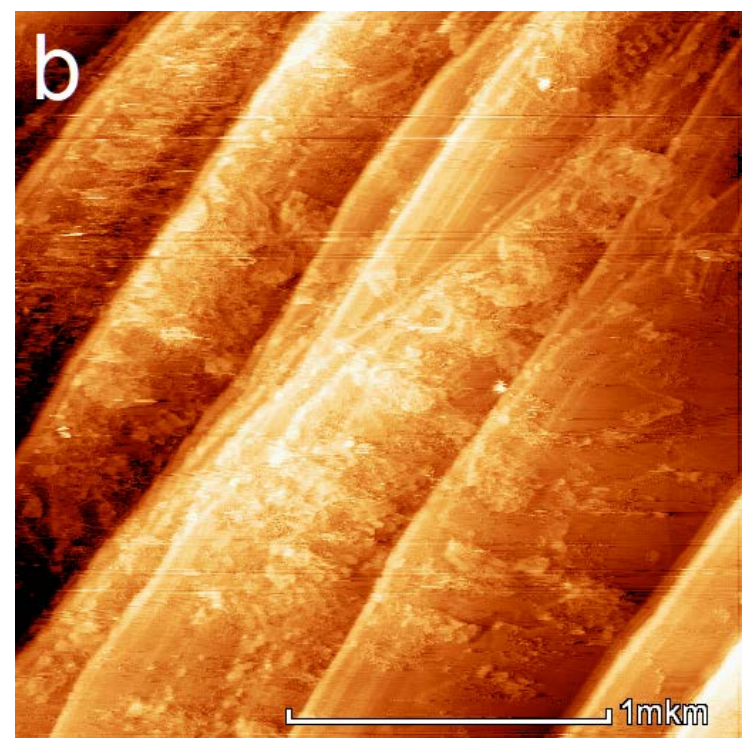

Fig. 4. STM images of ultra thin (a) and thin layers (b) of DPT-nAu on the surfaces of graphite

In this case, the width of the energy band $\left(E_{g}\right)$ at points on the surface, located at a distance of 200-300 nm, could vary substantially. For all LTCVCs, a nonlinear shape was found and was conformed the model of currents, limited by the space charge [18]. 


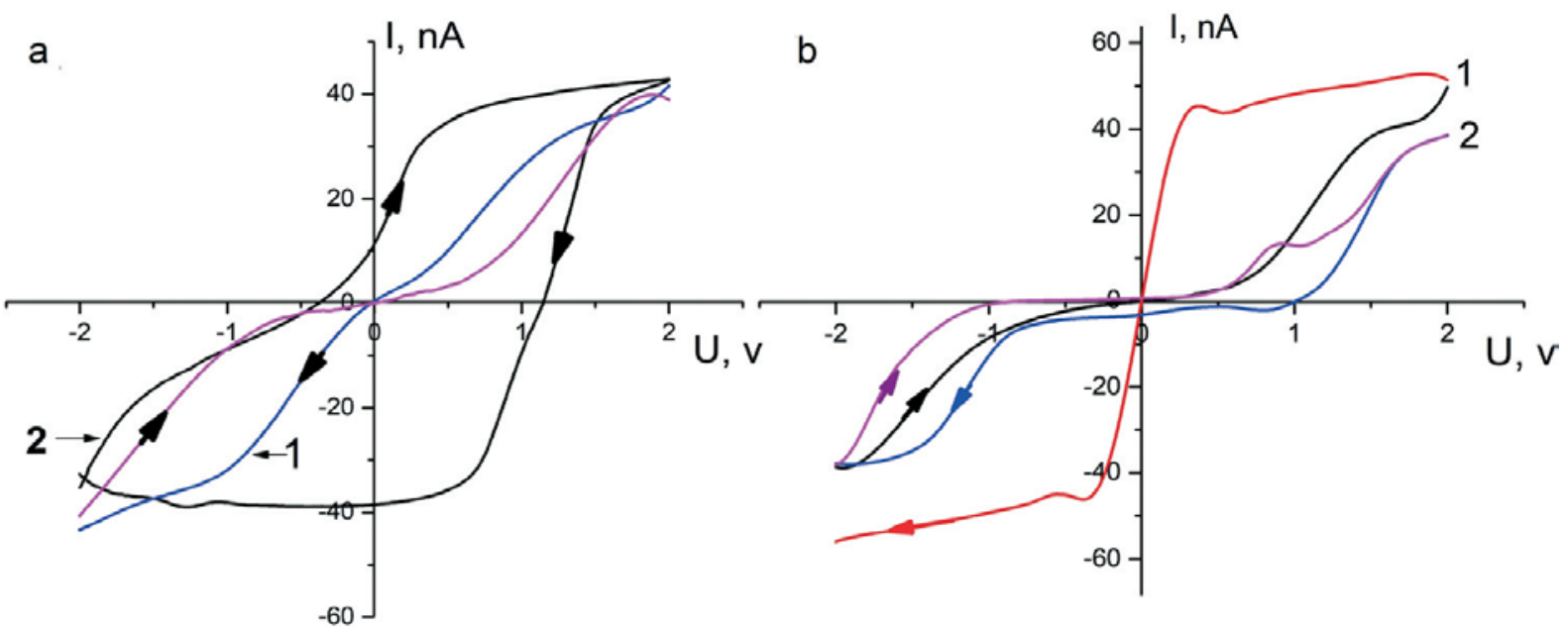

Fig. 5 . LTCVCs of DPT-0 (1) and DPT-1 (2) on the surfaces of gold (a) and graphite (b).

Regardless of the substrate and molecule charge, the effect of bipolar resistive switching was observed at all LTCVCs, i.e., the presence of areas of high (OFF) and low (SET) resistance, depending on the forward or reverse direction of the current. When a neutral molecule becomes an anion, the order of their succession also changes. Thus, the switching effect depends mainly on the charge of the DPT molecule, not on the substrate. The most interesting result was the presence of LTCVCs hysteresis or residual polarization in the DPT- 1 layers on the gold unlike crystallization of neutral PT molecules (Figure 5a). Perhaps, this indicates the formation of a ferroelectric. In the case of DPT-0 on the surface of gold, as well as DPT layers on graphite, hysteresis on the CVCs was not observed. The significant local heterogeneity of the electron energy structure of DPT layers was found. This is primarily associated with the chosen method of deposition. When measuring LTCVCs, a change in the thickness of DPT layers and their morphology can also lead to a change in the local strength of the electric field. The formed layers would have a local lateral disorder in both the interface region and the volume of the layer. In this case, the charge injection process and charge transport through the peptide layer also become laterally heterogeneous and it acquires a filamentary character [19]. The model of currents, limited by space charge, involves the electron capture by traps that arise in the dielectric layer in the contact region. Most of the applied voltage falls on the barriers due to the trapped surface states of the DPT, where the filling of which depends on the sign and the value of the applied voltage. As a result, a region of space charge can arise at the boundaries and in the volume of the DPT layer. When the polarity of the applied voltage is changed, the space charge region can expand or contract. This will change the number of the captured charge and the surface potential by the quantity of the captured charge potential. The microscopic nature of these traps is not clear and requires additional research. However, taking into account the data from [1], it can be assumed that they correspond to different functional groups in the DPT molecule. The more active interaction of DPT with gold in comparison with graphite can significantly influence both the morphology of the surface of the DPT layer and $\mathrm{E}_{\mathrm{g}}$ width. Therefore, on the inner boundary, the nature and energy of the surface states can also differ. Perhaps this is what we see when comparing LTCVCs on different substrates. From the data in Fig. 5, it is evident that, regardless of the substrate properties and the structure and morphology of the DPT layers, the decrease in positive charge of the DPT molecule with the increase in $\mathrm{pH}$ leads to a change in the sequence of the onset of the high-resistance and low-resistance states in the forward or reverse potential change. This behavior may be due to surface states localized at the outer boundary of the layer. In the absence of an external field, these states can be partially filled by the basic carriers.

The transport of charges through the DPT layer consists of successive jumps from molecule to molecule. In this case, they have to overcome the scattering of electrons due to intramolecular vibrations of traps that participate in the capture and emission of charges. This may be due to the polarization phenomena that are observed in dielectrics. Indeed, in the first case an organic crystal is formed, consisting of neutral DPT molecules, between which van der Waals forces act, and in the second, DPT salt, i.e., the ionic crystal. The mechanism of crystallization, the morphology, and the electrical characteristics of these two substances are significantly different. Therefore, when measuring the CVCs of the DPT- 1 layers, their polarization occurs, which changes sign when the polarity of the applied voltage is changed. An essential role is played also by the image charge on the gold surface and the associated potential of the formed dipole.

CVCs of peptide nanocomposite layers have much in common with CVCs DPT-0 and DPT-1. However, significant differences are also observed due to the presence of gold NPs (Figure 6). 


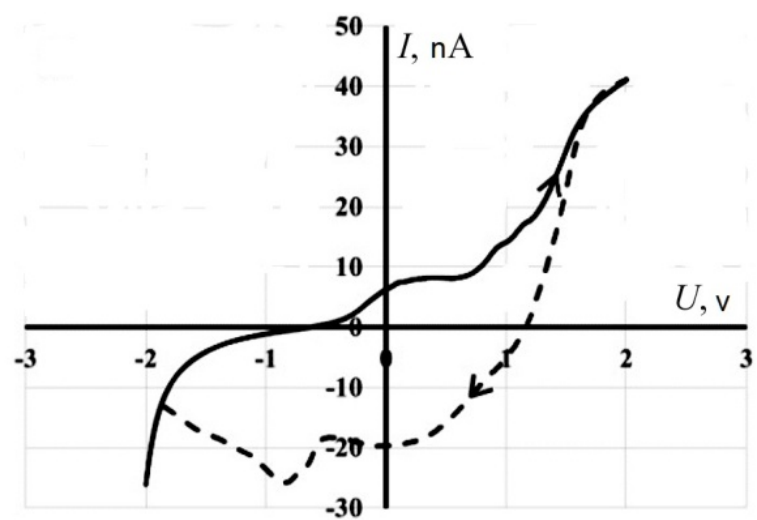

Fig. 6. LTCVCs of DPT-nAu on the surfaces of gold.

On LTCVCs of PT-Au composite, deposited on gold substrate, hysteresis similar to LTCVC of DPT-1 is also observed. However, besides this hysteresis the minimum of the current are observed. Such minimum has never been found in the case of DPT- 0 or DPT- 1 LTCVCs. The distance between minimum of current $\sim 1 \mathrm{eV}$ (Figure 7). With different samples of DPT-nAu composite on a gold substrate and at different points on the sample surface the position of these minimum, as well as their depth may vary. So, the second and other minimum of current at LTCVCs may be absent.

In contrast to DPT-nAu composite on gold LTCVCs on graphite always pass through 0 similarly to LTCVCs of DPT- 0 and DPT- 1 at graphite. At the same time, the minimum of current and bipolar resistive switching are observed both in thin DPT-nAu layers on graphite and gold.

It is interesting to compare LTCVCs of DPT-0, DPT1 и DPT-nAu layers on the surfaces of gold (Figure 7). It is clearly seen that the residual polarization depends on the negative charge of the DPT molecule. Indeed, it is absent in layers of neutral DPT molecules, but appears only when the molecule acquires a negative charge. In the synthesis of gold NPs, the total charge of the molecule decreases. This leads to a decrease in the residual polarization of the molecules. In its turn this can be explained if we take into account that the neutralization of ionized carboxyl groups on the surface of gold NPs leads to a decrease in the total negative charge of DPT anions and, therefore, to decrease $\mathrm{pH}$ of the solution. It is also necessary to take into account the interaction of DPTs with a gold substrate, which can also affect the trapped charge and therefore the features of LTCVC.

The results show significant dependence of the morphology of peptide composite layers from the nature of the substrate and the state of its surface. It indicates the significant role of interfacial interactions in the processes of their growth. Electrical properties of DPT-

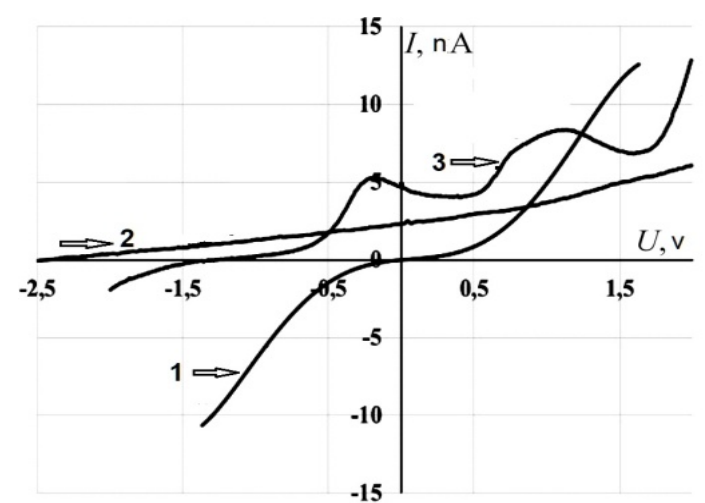

Fig. 7. LTCVCs of DPT-0 (1) DPT-1 (2) and DPT-nAu (3) on the surfaces of gold

nAu composite layers also depend on DPT interactions with the substrate. The increase of the layers thickness significantly affects both their morphology and tunneling current, i.e. by the amount of applied electric field. In electrical measurements, this leads to a change in the nature of LTCVC and, in particular, to the appearance or absence minimum of current on it.

As we have already noted, in LTCVCs of DPT-nAu composite several minima are visible. This is the region of negative differential conductivity (NDC). The appearance of a similar minimum at LTCVCs of composite materials based on cellulose derivatives and NPs of silver and gold was also observed [20].

According to the scheme at figure 8, the energy levels in the metal-peptide composite-metal structure with increasing of applied voltage gradually filled. So the appearance of different minimum of the tunneling current is observed. However, this scheme does not explain the cause of appearance of these levels and their capture of charge carriers. NDC is often observed when measuring the CVCs of various semiconductor inorganic materials, including composite ones (for example, Gunn, Esaki diodes, etc). However, in these cases, hysteresis is not observed on them. Probably the first who discovered the appearance of hysteresis on the CVCs were the authors of [21]. They pay attention to the similarity of measured CVCs with volt-ammograms, which are measured in electrochemistry. It has been suggested that the NDCs are due to electrochemical red-ox reactions that occur in strong electric fields of STM. Since by prolonged vacuum annealing of materials at high temperatures, the NDC disappeared, the authors suggested that this effect is due to the oxidation of water and the reduction of tungsten ions under the action of a strong electric field. It is well possible that we are also dealing with red-ox reactions in the peptide composite layer. This assumption is supported by the results of measurements of the IR spectra of the composite DPTnAu layer [6] 


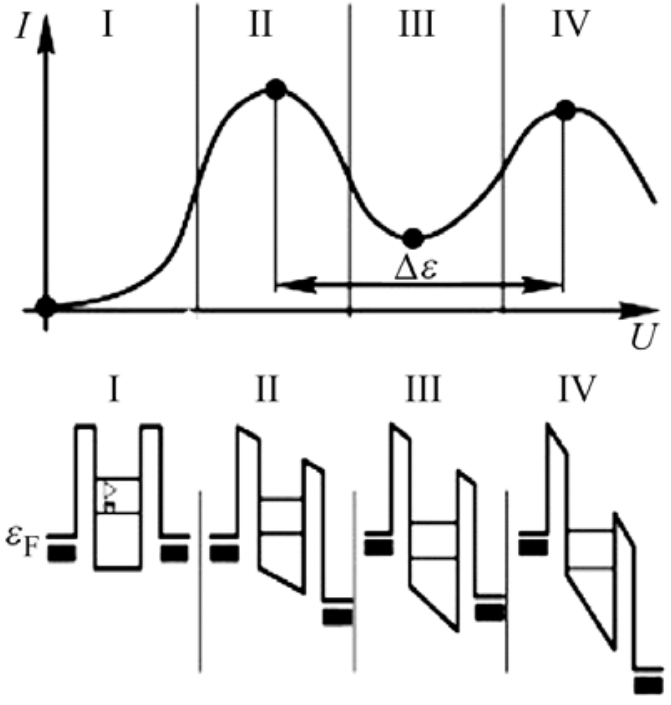

Fig. 8. Scheme of energy levels in structure metal-DPT composite-metal, illustrating the occurrence of negative differential conductivity.

During the synthesis of gold NPs in solution, stabilization of NPs surface occurs due to the adsorption of ionized carboxyl groups. Moreover, they may form various types of adsorption bonds with the surface of gold NPs (bridged, mono and bidentate, etc.). The formed Au-COO- bonds can create energy levels in the band gap of the peptide composite, which may capture charges. The filling of these levels depends on the sign and magnitude of the applied voltage. As already noted, the nature of CVCs of peptide layers is best consistent with the model of currents limited by the space charge. It involves the capture of electrons by traps that arise in the dielectric layer at the interfaces. As a result, a space charge region may appear at the interfaces and in the bulk of the Au-DPT layer. In this case, one must also take into account the surface states at the substrate-DPT$\mathrm{nAu}$ interface. When the polarity of the applied voltage changes, the space charge region may expands or contracts.

There may be another explanation for this effect. Given that gold NPs are in the form of flat disks with a height of $\leq 4 \mathrm{~nm}$, there is a possibility of dimensional quantization of the energy levels in height. For NPs, that are distributed along this height. Even if the distance between the levels in vacuum may seem too large $(\sim 1$ $\mathrm{eV})$, inside peptide matrix, this distance increases in proportion to the ratio of the effective masses of electrons in the NPs and matrix [20]. However, the absence of exact values of the effective masses of the electron in DPT does not allow the corresponding calculations and so to confirm or deny such possibility.

The most significant differences, in addition to the structure and morphology of layers, were also found in the shape of LTCVCs when comparing composite layers on graphite and gold. It follows that the interaction of the DPT with the substrate not only leads to a change in the conformation of the molecule in the adsorption layer [7], but also determines the final structure of DPT

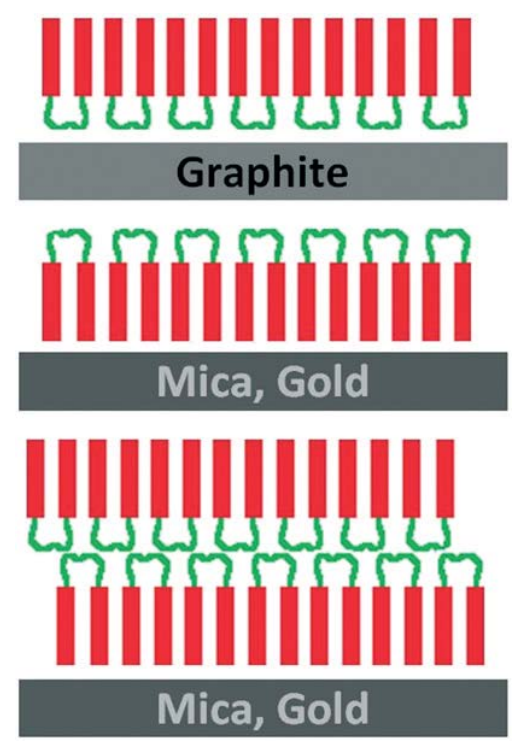

Fig. 9. Possible scheme of orientation of DPT monolayers on graphite, gold, and mica surfaces.

multilayers hundreds of nanometers thickness. It is a very interesting result that awaits its further study. Similar to solid DPT-0 and DPR-1 layers, the effect of bipolar resistive switching is also observed in DPT-nAu layers. It can find practical application in the manufacture of DPT memristors.

The materials we studied differed not only in their structure and chemical composition, but also in the surface charge. Indeed, the surfaces of graphite is electrically neutral, and on the surface of gold a positive charge arises only when DPT-1 is adsorbed (electric image force). Therefore, when DPT-0 and DPT- 1 are deposited on the surfaces of these materials, the nature of their interaction will be significantly different. Among all the substrates studied, the surface of graphite is the most inert and hydrophobic. After DPT deposition, it becomes hydrophilic in contrast to gold,

Previously, the scheme of their orientation in the adsorption layer was proposed [7]. This scheme diagram is shown in Figure 9. From this scheme, it follows that the functional groups of PTs (antenna) are oriented perpendicular to the surface of the mica and gold. Wherein, the hydrophobic bridges form the outer layer of the adsorbate. Differences in the nature of adsorbateadsorbent interactions on the surface of gold and graphite lead to differences in the growth lead to a significant change in the electrical properties of solid layers, for example, the appearance of residual polarization in DPT-nAu and DPT-1 on the surface of gold and its absence on the surface of graphite.

\section{Conclusion.}

The results demonstrate that the structure and morphology of DPT thin layers depend significantly on the molecule charge (neutral or anion) and the nature of the substrate-layer interface. It was possible to control the structure and properties of the formed solid layers by 
changing the $\mathrm{pH}$ of the solution (the charge of the DPT molecule). Bipolar resistive switching was observed in thin peptide layers on graphite and gold surfaces. The crystallization of anions on the surface of gold unlike graphite led to the formation of a ferroelectric.

A strong dependence of the morphology of the DPT composite layers on the nature of the substrate and the state of its surface is revealed. It indicates the important role of interfacial interactions in the crystallization processes of the DPT layers. The electrical properties of layers also depend on the interaction of DPT with the substrate. An increase in the thickness of the layers significantly affects the morphology and value of the tunneling current. Similar to crystallization of the salt of the peptide on a gold surface, crystallization of DPT$\mathrm{nAu}$ composite also leads to the formation of a ferroelectric. Such differences in the structure of composite layers on graphite and gold surfaces can be explained if we assume that the structure of the second and all subsequent layers is completely determined by the structure of the first adsorption layer in DPTsubstrate interface. So this layer serves as a template for the growth of all other layers.

The results can find practical application in 3D printing technologies. The presence of NDC on CVCs of peptide composites is of great practical importance when used as active elements for amplifying current and power, memory cells. Investigated DPT has rather good memristive characteristics, including good endurance, satisfying ON/OFF current ratio, long retention time and reproducible write-once read-many times (WORM) memory behavior. All this allows us to consider the DPT as a perspective material of memristor organic electronics. Since it is also a drug, the polymorphism and its dependence on $\mathrm{pH}$ can also find application in the pharmaceutical industry.

Acknowledgements. This study was supported by the Ministry of Education and Science of the Russian Federation grant 9.1195.2017/4.6. We also thank the Center of Collective Use of MSUT "STANKIN" for providing resources.

\section{References}

1. C. Mukherjee, M.K. Hota, D. Naskar, S.C. Kundu, C.K. Maiti, Phys. Status Solidi A 210, 1797, (2013)

2. J. Ouyang, C.-W. Chu, C.R. Szmanda, L. Ma, Y. Yang, Nat. Mater. 3, 918 (2004)

3. J.C. Chen, C.L. Liu, Y.S. Sun, S.H. Tung, W.C. Chen, Soft Matter, 8526 (2012)

4. A.I. Loskutov, O.Ya. Uryupina, S.N. Grigor'ev, V.B. Oshurko and V.I. Roldughin, Colloid J., 75(3), 301 (2013)

5. A.I. Loskutov, O.Y. Uryupina, S.N. Grigor'ev, N.V. Kosheleva, V.B. Oshurko, E.V. Romash, I.N. Senchikhin and A.V. Falin, Prot. Met. Phys. Chem. Surf., 51, 558 (2015)

6. A.I. Loskutov, O.A. Guskova, S.N. Grigor'ev, V.B. Oshurko,A.V. Tarasiuk and O. Ya. Uryupina, J. Nanopart. Res., 18, 238, (2016)
7. A. Loskutov, B. Lokshin, T. Gudasheva, V. Oshurko, N. Solis Pinargote, N. Sazonova and V. Romanov, Cryst. Eng. Comm, 20, 7688 (2018)

8. B.A Grzybovski, Cryst Eng Comm., 16, 9368 (2014)

9. E. Linn, R. Rosezin, C. Kugeler, R. Waser, Nat. Mater. 5, 403 (2010)

10. R. Waser, M. Aono, Nat. Mater. 6, 833 (2007).

11. S. Kumar, J.P. Strachan and R.S. Williams, Nature, 548, 318 (2017)

12. G.D. Zhou, Z. Ren, L. Wang, J. Wu, B. Sun, A. Zhou, G. Zhang, S. Zheng, S.K. Duan and Q.L. Song, Nano Energy., 63, 103793 (2019)

13. Q. Xia, J.J. Yang, Nat. Mater., 18, 309 (2019)

14. C.O. Callaghan, C.G. Rocha, F. Niosi, H.G. Manning, J.J., Boland and M.S. Ferreira, J. Appl. Phys., 124, 152118 (2018)

15. T. You, L.P. Selvaraj, H. Zeng, W. Luo, N. Du, D. Bürger, I. Skorupa, S. Prucna, A. Lawerenz, T. Mikolajick, O.G. Schmidt and H. Schmidt, Adv. Electron. Mater., 2, 1500352 (2016)

16. S. Seredenin, T. Gudasheva, Dipeptide mimetics of neurotrophines NGF and BDNF, US Pat., 9683014B29 (2017)

17. R.D. Deegan, O. Bakajin, T.F. Dupont, G. Huber, S.R. Nagel and T.A. Witten, Phys. Rev. E: Stat. Phys., Plasmas, Fluids, Relat. Interdiscip. Top., 62, 756 (2000)

18. M.A. Lampert and P. Mark, Current Injection in Solids (Academic Press, New York, 1970)

19. J.J.M. van der Holst, F.W.A. van Oost, R. Coehoorn and P.A. Bobbert, Phys. Rev. B: Condens. Matter Mater. Phys., 80, 235202 (2009)

20. O.Ya. Uryupina, V.V. Vysotskii, A.I. Loskutov, A.V. Cherkasova, and V.I. Roldugin, Rus. J. of Appl. Chem., 86(8), 1268-1274 (2013)

21. D. Azulay, F. Kopnov, R.T. Reshef, I. Balberg, O. Millo, Nano Lett., 6, 760 (2006) 